- RAM, REV. ADM. MACKENZIE, V. 12, N. 3, Edição Especial • SÃO PAULO, SP • MAIO/JUN. 2011 • ISSN 1678-6971 • Submissão: 17 jan. 2011. Aceitação: 18 mar. 2011. Sistema de avaliação: às cegas dupla (double blind review). UNIVERSIDADE PRESBITERIANA MACKENZIE. Walter Bataglia (Ed.), p. 177-206.

\title{
UMA COMPREENSÃO DA SUSTENTABILIDADE POR MEIO DOS NÍVEIS DE COMPLEXIDADE DAS DECISÕES ORGANIZACIONAIS
}

\section{ANDREI GIOVANI MAIA}

Doutor em Administração e Turismo pelo Programa de Pós-Graduação em Administração e Turismo da Universidade do Vale do Itajaí (Univali).

Professor do Departamento de Administração do Instituto de Ensino Superior Santo Antônio (Inesa).

Rua Papa João XXIII, 1.100, Iririú, Joinville - SC - Brasil - CEP 89227-301

E-mail: andreigm@ig.com.br

\section{PAULO DOS SANTOS PIRES}

Doutor em Ciências pela Faculdade de Filosofia, Letras e Ciências Humanas da Universidade de São Paulo (USP). Professor do Programa de Mestrado em Turismo e Hotelaria da Universidade do Vale do Itajaí (Univali).

Univali, Campus Balneário Camboriú, bloco 7, sala 205/206, Municípios, Balneário Camboriú - SC - Brasil - CEP 88337-300 


\section{RESUMO}

Em uma sociedade capitalista, as decisões organizacionais, geralmente voltadas à dimensão econômica, desencadearam ou agravaram os problemas socioambientais em muitos países. Na busca por soluções de tais problemas, surge, na década de I980, o conceito de desenvolvimento sustentável, propondo o atendimento das necessidades das gerações atuais sem comprometer o atendimento das necessidades das futuras gerações (COMISSÃO MUNDIAL SOBRE MEIO AMBIENTE E DESENVOLVIMENTO, I988). Considerado um conceito subjetivo e passível de interpretação, o desenvolvimento sustentável propõe o atendimento de três dimensões essenciais: econômica, social e ambiental. Assim, sob a ótica organizacional, tomar decisões que contribuam para a sustentabilidade tornou-se, no mínimo, um grande desafio, pois, além da dimensão econômica, os decisores deverão lidar simultaneamente com as dimensões sociais e ambientais. Isso mostra a existência de níveis de decisões definidos como simples, medianos e complexos, os quais tendem a comprometer ou influenciar de forma distinta o alcance da sustentabilidade, conforme o atendimento satisfatório ou não das dimensões. Diante desse desafio, como ensaio teórico-metodológico, este artigo tem como objetivo apresentar uma compreensão da sustentabilidade por meio dos níveis de complexidade das decisões organizacionais. Para alcançar esse objetivo, propõem-se critérios relevantes que compõem cada uma das dimensões da sustentabilidade, identificando as relações (sistêmicas) que podem ocorrer entre os critérios durante a tomada de decisões organizacionais. Como resultado do cruzamento entre níveis de complexidade de decisões e as dimensões da sustentabilidade, obteve-se a estrutura de uma matriz de complexidade das decisões direcionadas à sustentabilidade. De caráter qualitativo, a matriz visa mostrar como o decisor ou pesquisador pode classificar as decisões consideradas relevantes e como poderá tomar decisões complexas que integrem satisfatoriamente as três dimensões da sustentabilidade. 


\section{PALAVRAS-CHAVE}

Sustentabilidade; Dimensões da sustentabilidade; Critérios das dimensões; Níveis de decisões; Organizações.

\section{INTRODUÇÃO}

A história da humanidade foi moldada pelas decisões tomadas por indivíduos, civilizações e nações na busca por sua sobrevivência ou domínio. Com o passar dos séculos, as organizações produtivas passaram a dominar e a influenciar as sociedades, e suas decisões tiveram sérias implicações ambientais e sociais. O sistema capitalista de produção, durante os últimos dois séculos, estimulou a concentração da riqueza, o consumo e a exploração dos recursos naturais pelas organizações de forma intensa e, via de regra, desordenada, gerando problemas sem precedentes.

Diante dos problemas ambientais e sociais desencadeados pelas decisões tomadas dentro e fora das organizações, surge o desafio de alcançar a sustentabilidade, considerando essencialmente três dimensões do desenvolvimento sustentável: econômica, social e ambiental (COMISSÃO MUNDIAL SOBRE MEIO AMBIENTE E DESENVOLVIMENTO, I988; DIAS, 2008; CIEGIS; RAMANAUSKIENE; MARTINKUS, 2009), também conhecidas como triple bottom line, nos termos de Elkington (2004). A busca pelo equilíbrio entre as dimensões econômica, social e ambiental passa, então, a fazer parte do discurso sobre a sustentabilidade.

Considerando que muitos problemas socioambientais foram causados porque decisões simples e fragmentadas visavam atender apenas à dimensão econômica, dentro do conceito de homem econômico, defendido pelos pensadores clássicos da Administração ou, ainda, de homem administrativo, estudado por Simon (I97I), aponta-se para a necessidade de decisões complexas que busquem no mínimo o equilíbrio entre as dimensões econômica, social e ambiental simultaneamente.

A tomada de decisões direcionadas à sustentabilidade exige do decisor a capacidade de lidar com múltiplas variáveis e dimensões de forma simultânea, juntamente com os problemas desestruturados de difícil definição. Nesse sentido, o pensamento sistêmico soft surge como uma abordagem desenvolvida justamente para lidar com situações de elevada complexidade (CHECKLAND, 2000).

Assim, o objetivo deste ensaio teórico, elaborado a partir de tese de doutorado defendida e aprovada em outubro de 20ı, é apresentar uma compreensão da 
sustentabilidade por meio dos níveis de complexidade das decisões organizacionais. Para alcançar esse objetivo, o trabalho abordou teoricamente a sustentabilidade, suas dimensões e seus critérios, assim como as decisões organizacionais e seus níveis de complexidade. Com base nesse referencial teórico, foi possível propor a estrutura de uma matriz que relaciona as dimensões da sustentabilidade com os níveis de decisões que se aplicam às organizações, permitindo classificar as decisões por meio do atendimento dos critérios de cada dimensão.

\section{REFERENCIAL TEÓRICO}

\subsection{O DESAFIO DA SUSTENTABILIDADE PARA O SÉCULO XXI}

Durante o século XX, os impactos provenientes principalmente da pressão industrial, das forças de mercado sobre o ambiente natural, da concentração de capital nas mãos de poucos e do crescimento populacional deflagraram crises extremamente complexas de serem resolvidas.

Diante dos excessos do sistema capitalista de produção sobre o ambiente natural e ante os impactos negativos na sociedade, percebidos por muitas nações, as conferências da Organização das Nações Unidas (ONU), realizadas em I972, em Estocolmo, na Suécia, e em i982, em Nairóbi, no Quênia, marcaram os debates que levariam ao conceito de desenvolvimento sustentável, em I987, com a publicação do Relatório Brundtland ou Nosso futuro comum, pela Comissão Mundial sobre Meio Ambiente e Desenvolvimento (Cmmad).

Segundo a Comissão Mundial sobre Meio Ambiente e Desenvolvimento (I988, p. 44), “o desenvolvimento sustentável procura atender às necessidades e aspirações do presente sem comprometer a possibilidade de atendê-las no futuro". O conceito foi disseminado posteriormente, com a Conferência da Organização das Nações Unidas (ONU), realizada no Rio de Janeiro, em I992 (Rio 92), e a partir daí o interesse sobre o assunto cresceu e se globalizou.

Para Jeffrey Sachs (2008, p. I3), "os desafios do desenvolvimento sustentável - protegendo o meio ambiente, estabilizando o crescimento demográfico mundial, reduzindo as diferenças entre ricos e pobres e acabando com a miséria - tomarão o centro do palco". Segundo Sachs (2008), as quatro principais causas das crises sociais e ecológicas mundiais a serem enfrentadas pela humanidade são: as pressões humanas sobre os ecossistemas e o clima da Terra; o crescimento da população mundial; a miséria e a pobreza não aliviadas pelo crescimento econômico; o peso do cinismo, do derrotismo e de instituições ultrapassadas na insolubilidade dos problemas globais. 
Diante dessas quatro causas a serem enfrentadas, Sachs (2008) salienta que as soluções para os problemas sociais e ecológicos não estão nas forças de mercado, tendo em vista sua incapacidade de superá-los, mas sim na cooperação global.

De acordo com Guimarães (2006, p. 29), "a evolução da percepção sobre os desafios atuais, manifestada também nas ações concretas que levaram à 'nova' agenda global, permite uma avaliação positiva a respeito do contexto internacional para o desenvolvimento sustentável".

Ao se reconhecerem as necessidades de diversas nações, constata-se que a construção de uma sociedade sustentável é, no mínimo, um grande desafio para o século XXI. As decisões e ações da humanidade em relação à sustentabilidade determinarão seu futuro e o das próximas gerações. Este é um momento de busca por soluções conscientes e sistêmicas que reconheçam a complexidade dos problemas ambientais e sociais. Além disso, deixar a responsabilidade pelas soluções dessas crises somente nas mãos de políticos ou da própria força de mercado não parece uma alternativa viável, pois, conforme Guimarães (2006), os países desenvolvidos buscam defender seus próprios interesses.

Ainda segundo Sachs (2008), as tecnologias sustentáveis podem contribuir muito para a superação dessas crises e suas causas, convergindo com os argumentos de Capra (2005), ao salientar que a construção de uma sociedade sustentável para as futuras gerações depende de uma revisão das tecnologias e das instituições sociais, na tentativa de superar o distanciamento entre projetos humanos e sistemas ecologicamente sustentáveis da natureza.

Contudo, é importante frisar que as tecnologias mais recentes têm contribuído para o crescimento econômico e o bem-estar social em curto prazo, mas também têm gerado problemas ambientais e sociais de longo prazo sem precedentes.

Conforme Guimarães (2006, p. 52), "as possíveis soluções à atual crise de civilização através do desenvolvimento sustentável haverá que buscá-las no próprio sistema social, e não em alguma mágica tecnológica ou de mercado".

Apesar do reconhecimento da importância dessas perspectivas sobre a tecnologia, existe um elemento que deve estar na essência das decisões e ações humanas: a consciência ecológica. Para que se chegue a esse nível de consciência, a educação em relação à sustentabilidade é fundamental.

A consciência humana pode influenciar o desenvolvimento de tecnologias que visem à sustentabilidade. Contudo, apostar somente no desenvolvimento de novas tecnologias para o alcance da sustentabilidade soa como uma medida que visa apenas prorrogar os inevitáveis problemas ambientais e sociais. Além da necessidade de estender o desenvolvimento de tecnologias a toda a população, as decisões conscientes devem vir acompanhadas da redução no ritmo destrutivo da atividade humana sobre a natureza e da coerente redução na taxa do crescimento populacional. 
De acordo com Sachs (2008), as forças de mercado, sozinhas, não têm condições de solucionar esses problemas, pois: I. não podem garantir o desenvolvimento de tecnologias sustentáveis, mesmo porque essas tecnologias seriam direcionadas aos benefícios sociais e não à lucratividade; 2. mesmo que sejam desenvolvidas, não existem garantias de sua ampla adoção, pois tais tecnologias precisam geralmente de incentivos e estímulos para sua aceitação; 3. porque não podem garantir as mudanças populacionais necessárias, seja em um país, seja em nível global; 4. finalmente, as forças de mercado não poderão assegurar o atendimento das necessidades básicas em todas as partes do mundo, na medida em que seguirem um rumo de crescimento convergente.

Isso já havia sido previsto pelo economista ecológico Herman Daly (I99I, p. I2), ao salientar o seguinte: “os que quiserem beneficiar-se da 'mão invisível' do mercado, ainda que maravilhosa para realizar a alocação, não tem capacidade de determinar nem a escala (da economia), nem a distribuição ótima (dos recursos)". Portanto, a suposição de que as forças de mercado serão capazes de superar os problemas (crises) sociais e ecológicos causados pelo ritmo acelerado imposto por essas mesmas forças se evidencia como um paradoxo.

Para Sachs (2008, p. 47), “desenvolvimento sustentável significa prosperidade globalmente compartilhada e ambientalmente sustentável”. Assim, para o desenvolvimento sustentável, são necessárias três mudanças fundamentais: sustentabilidade ambiental, estabilização populacional e fim da miséria (SACHS, 2008). E essas mudanças só poderão ser alcançadas com uma mobilização global, fundamentada em um processo de cooperação e interação entre povos, mas que exigirá negociação e acomodação entre as visões de mundo criadas pelas pessoas, regiões e nações sobre a sustentabilidade.

O conceito de sustentabilidade deve ser uma construção consensual entre indivíduos, organizações e nações, pois, como afirma Capra (2006), as pessoas não são resistentes às mudanças, são resistentes, sim, às mudanças que a elas são impostas.

\subsection{AS TEORIAS ECONÔMICAS ACERCA DA SUSTENTABILIDADE}

Com a repercussão das problemáticas ambientais emergidas nas décadas de I960 e I970, ocorreu uma forte influência do ambientalismo nos estudos científicos que incorporaram a perspectiva ecológica e ambiental em seus enfoques. As pesquisas econômicas passaram, então, a receber influência ambientalista, fazendo surgir duas importantes perspectivas teóricas, direcionadas às propostas de desenvolvimento sustentável: a economia ecológica e a economia ambiental (ecossocioeconomia). 
A economia ecológica é representada por economistas como Kenneth Eward Boulding, Karl-William Kapp, Nicholas Georgescu-Roegen, Herman E. Daly, Robert Costanza, Juan Martínez-Alier, pelo teólogo John Cobb Jr., pelo ecologista Crawford Stanley Holling, pelo ambientalista Lester Brown, além de economistas brasileiros como Clóvis Cavalcanti, José Eli da Veiga e Ladislau Dowbor.

Em linhas gerais, a economia ecológica propõe "um enfoque multidisciplinar para a gestão do meio ambiente, excluindo o crescimento quantitativo de seus pensamentos a favor de um desenvolvimento qualitativo" (MORALEJO; MIGUEL; LEGARRETA, 2009, p. IO2). O desenvolvimento sustentável é para a economia ecológica a resposta à crise ambiental gerada pelo paradigma economicista e excludente, atualmente dominante (MONTIBELLER FILHO, 200I).

Os pressupostos da economia ecológica se fundamentam nas leis da termodinâmica. A primeira lei da termodinâmica, também conhecida como princípio da conservação de energia, afirma que a energia (ou matéria) não pode ser criada ou destruída, pode apenas ser transformada em outra forma.

No contexto da economia ecológica, Daly (I99I) explica que tanto a retirada de matéria e energia como sua inserção em um ecossistema devem romper o funcionamento normal deste, ou seja, tanto a ausência como o excesso de matéria e energia causarão um efeito no ecossistema.

Como afirma Bertalanffy (2008, p. 67), "já foi dito que a energia é a moeda corrente da física, assim como os valores econômicos podem ser expressos em dólares ou libras".

A segunda lei da termodinâmica afirma que a matéria-energia transformada para realizar certo trabalho útil resulta parcialmente em outra forma de energia, pois, durante o processo de transformação da energia, parte não é aproveitada, ou seja, degrada-se. Em relação à segunda lei da termodinâmica, Daly (I99I, I996) expõe que há uma diferença qualitativa entre a matéria-energia retirada satisfazer as necessidades humanas, que é de baixa entropia, e aquela reinserida pelo homem na forma de resíduos de alta entropia.

Então, tem-se outro enunciado da segunda lei da termodinâmica em relação à ordem e à desordem da matéria e energia: em processos espontâneos, a ordem tende a transformar-se em alta ou baixa desordem (entropia), sendo muito improvável reverter tal processo (princípio da irreversibilidade) (TEIXEIRA JUNIOR, I982).

Além das leis da termodinâmica, os economistas ecológicos fazem referência a uma distinção sugerida por Boulding (ig66) entre dois tipos de economias: "a economia do cowboy" e a "economia do astronauta". A economia que depende essencialmente do ambiente natural para a sobrevivência dos seres humanos, com pouco uso de tecnologias e reduzida exploração dos recursos naturais, é denominada economia do cowboy. A economia que depende da tecnologia para a 
sobrevivência dos seres humanos, obviamente com elevadas tecnologias e intensa exploração dos recursos naturais, é classificada como economia do astronauta.

Daly (I99I, p. I0) argumenta que, "em um sistema finito, sujeito à conservação de matéria-energia, quanto maior a parte que for posta sob nosso controle econômico, menor será a parte que permanece sob controle espontâneo da natureza”.

Desse modo, segundo Daly (I99I), a maior parte das nações estaria entre as economias do cowboy e do astronauta, e essas nações deveriam considerar as leis da termodinâmica em seu processo de uso da matéria-energia. A escolha do tipo de economia pode determinar o colapso do ambiente natural e seu consequente impacto na economia e na sociedade.

Diante desse conflito, Brown (2003) sugere que, para uma economia ambientalmente sustentável, ou seja, uma ecoeconomia, é necessário que os princípios da ecologia fundamentem a formulação de políticas econômicas e que tanto economistas quanto os ecólogos trabalhem juntos com o objetivo de modelar a nova economia.

Em seu trabalho, Boulding (I966, p. 6) já havia alertado sobre os riscos de a humanidade tentar pilotar "a espaçonave Terra" por meio da economia do astronauta, ao destacar que "a sombra da futura espaçonave, na verdade, já está se projetando sobre a nossa alegria esbanjadora. Estranhamente, parece ser na poluição, mais do que na exaustão, que o problema está se tornando saliente [...]".

Outra questão discutida no âmbito da economia ecológica é a distinção entre o conceito de desenvolvimento e de crescimento econômico. Kapp (I976) é um dos precursores da crítica ao crescimento econômico para a resolução dos problemas dos países atrasados. Ele argumenta que um ambiente de crescimento econômico agrava a degradação ambiental e gera consequências desfavoráveis e inibidoras do desenvolvimento nos países mais atrasados.

A economia de mercado é, para Kapp, um "sistema de custos não pagos", geradora de elevados custos sociais e de degradação ambiental (LUZZATI, 2005). Para Kapp (I976), o problema-chave é considerar a economia como um sistema aberto, em um planeta que, segundo Boulding (I966), é um sistema fechado (finito). Portanto, a posição de Kapp é de que as necessidades sociais desejáveis devem ser atendidas, mantendo o estado dinâmico de equilíbrio ecológico e econômico (LUZZATI, 2005). Esse posicionamento de Karl William Kapp influenciou a economia ecológica, pois, para os economistas ecológicos, crescimento significa, em sua essência, aumento quantitativo, diferentemente do termo desenvolvimento, que está relacionado à melhoria qualitativa (DALY, I99I, I996). Assim, para Daly (I99I, p. I4), “desenvolvimento sustentável é uma melhoria qualitativa que não implique um aumento quantitativo maior do que o aceitável pela capacidade de suporte [...], é desenvolvimento sem crescimento". 
Na compreensão dos economistas ecológicos, a busca por um crescimento contínuo das economias mundiais, para o aumento de suas riquezas, vai torná-las, no futuro, de fato, mais pobres e não mais ricas, ou seja, na atual condição, o crescimento trará maiores custos do que benefícios, podendo ainda levar à superação da escala ótima (DALY, I99I). A suposição capitalista de que o capital é um substituto perfeito para os recursos naturais é uma perspectiva inconsistente, pois, segundo Daly (I991, I996), capital é um recurso complementar ao natural, e não um substituto perfeito. Nesse caso, observa-se que a economia ecológica é caracterizada como uma moderna versão neomaltusiana que alerta para o crescimento populacional e para o uso desregrado dos recursos do planeta.

A tecnologia é outro enfoque discutido pelos economistas ecológicos, pois, para a maximização do capital, a adoção de tecnologias vem provocando a intensificação do uso dos recursos naturais. Daly (I99I) argumenta que os hábitos relacionados ao uso de tecnologia devem ser redirecionados para maximizar a produção dos recursos naturais, que representam o principal fator limitante. Para Daly (I99I, I996), a economia é apenas um subsistema aberto de um sistema total finito e fechado, sendo necessário definir a escala ótima da economia. Assim, a publicação de The limits to growth [Os limites do crescimento] e Beyond the limits [Além dos limites] abalaram a convicção dos otimistas tecnológicos.

Meadows, Meadows e Randers (I992 apud GOODLAND, I995, p. 8) concluem que "é possível alterar a tendência de crescimento e estabelecer uma condição de estabilidade ecológica e econômica que é sustentável para o futuro".

Os economistas ecológicos definem como princípios para a sustentabilidade: I. a necessária limitação escalar da economia humana, respeitando os limites de suporte natural; 2. o avanço técnico deve ser direcionado à maximização da eficiência, e não da degradação do ambiente natural; 3. os recursos naturais renováveis não devem ser levados à extinção pela maximização produtiva, buscando vincular as colheitas ao ritmo de regeneração e à emissão de resíduos à assimilação do ambiente; 4 . os recursos renováveis devem ser criados para substituir no mesmo ritmo os da exploração dos não renováveis (DALY, I99I, I996).

Apesar do arcabouço teórico que delineia o pensamento da economia ecológica, nem sempre há pleno consenso entre os economistas ecológicos em relação a outros assuntos que tratam da sustentabilidade. O pluralismo está presente nas percepções e interpretações individuais e é reconhecido como essencial para a economia ecológica. Conforme Amazonas (2009), a economia ecológica não é uma teoria pronta e claramente definida, mas, sim, uma perspectiva interdisciplinar de saberes que busca compreender a relação entre economia e ecologia e que possui como fundamento o pluralismo metodológico.

Dessa forma, as diversas interpretações sobre sustentabilidade viriam a agregar ainda mais para a evolução da economia ecológica, pois essa corrente 
aceita a perspectiva de outras visões de mundo ou paradigmas. Não se caracteriza como uma teoria excludente, mas, sim, como uma teoria de compreensão das visões de mundo sobre atitudes ecológicas conscientes em relação ao desenvolvimento sustentável.

Por sua vez, a economia (sócio) ambiental é representada pelos economistas Ignacy Sachs (ecossocioeconomia), Salah El Serafy e Jeffrey Sachs, e pelo ambientalista Maurice Strong, além dos representantes brasileiros, como o filósofo Paulo Henrique Freire Vieira. Essa corrente teórica da sustentabilidade, de postura neoliberal, tem seus fundamentos originados nos debates seminais promovidos pela Organização das Nações Unidas (ONU), por intermédio da Conferência de Estocolmo (Suécia, I972) ou sob a influência das diretrizes do Relatório Brundtland, publicado em I987, pela Comissão Mundial sobre o Meio Ambiente e Desenvolvimento (Cmmad).

A economia ambiental "tem por objetivo alcançar a sustentabilidade dos recursos naturais por meio da tecnologia [...]" (MORALEJO; MIGUEL; LEGARRETA, 2009, p. IO०). Com os estudos de Ignacy Sachs e sua equipe interdisciplinar do Centro Internacional de Pesquisas sobre o Meio Ambiente e o Desenvolvimento (Cired), emerge o conceito de ecodesenvolvimento, que passou, mais tarde, a ser reconhecido pelo próprio Sachs como sinônimo de desenvolvimento sustentável.

Conforme Vieira (2007), um dos principais representantes dos postulados de Ignacy Sachs no Brasil, o conceito de ecodesenvolvimento indicava um novo estilo de desenvolvimento fundamentado no planejamento participativo e nos aspectos éticos de atendimento das necessidades humanas fundamentais e de consciência ecológica das sociedades.

Vieira (2007) sustenta seu enfoque na prevenção e na adaptação do planejamento local e territorial, para a aplicação nos sistemas complexos, dentro dos moldes da pesquisa-ação de corte sistêmico ou da gestão integrada e participativa dos recursos naturais.

Ao prefaciar o livro de Sampaio (2002), Vieira enfatiza ainda que o ecodesenvolvimento (desenvolvimento sustentável) representa uma modalidade de política ambiental que envolve simultaneamente prevenção e proatividade, fundamentada na integração interdisciplinar e transdisciplinar do conhecimento científico, proposta pela moderna teoria ecológico-humana. Assim, "a definição e a implementação das estratégias redundam no planejamento participativo, em que a participação da população é fundamental para o sucesso da estratégia escolhida" (DONAIRE, I999, p. 4I).

O planejamento participativo local traz, em seu arcabouço, a proposta de contribuir para o desenvolvimento sustentável dentro de certo território ou localidade, sempre com a participação da comunidade. Os economistas ambientais 
fundamentam a abordagem do planejamento local em ecoestratégias de desenvolvimento classificadas dentro das dimensões da sustentabilidade, sendo atualmente um total de oito dimensões definidas por Sachs (2002).

Além de apostarem na incorporação das dimensões da sustentabilidade para a resolução de problemas ecossocioambientais, os economistas ambientais também apostam que as tecnologias sustentáveis serão capazes de corrigir os graves problemas ambientais e que o crescimento econômico é necessário para ajustar as extremas disparidades entre os países ricos e pobres.

De acordo com os economistas ambientais, a tecnologia sustentável seria a grande saída para os problemas relacionados à insustentabilidade de economias capitalistas em estado avançado de exaustão de recursos naturais, representado no tipo de economia do astronauta.

Segundo Jeffrey Sachs (2008, p. 49), "para cada dimensão do desenvolvimento sustentável existe uma necessidade tecnológica fundamental, que deve ser calcada em investimentos na ciência básica".

Donaire (I999) destaca que as soluções propostas pelo ecodesenvolvimento estão relacionadas à correção específica do estilo de desenvolvimento de cada região, reconhecendo fatores culturais e ecológicos, além das necessidades de curto e longo prazo.

Em relação aos aspectos interpretativos da sustentabilidade, Vieira, ao prefaciar o livro de Sampaio (2002), reconhece as incertezas científicas, o inevitável pluralismo de visões de mundo, os sistemas éticos e as ideologias sociopolíticas. Neste mesmo prefácio, Vieira salienta que a criação de um sistema de gestão ambiental deve sustentar-se nos instrumentos integradores e de descentralização (SAMPAIO, 2002).

Essa visão integradora e descentralizada representa um instrumento heurístico que permite abordar os problemas relacionados ao desenvolvimento de forma mais ampla, envolvendo tanto a ecologia natural quanto a ecologia cultural (SAMPAIO, 2002).

A teoria da economia ambiental traz importantes contribuições às estratégias de desenvolvimento local, principalmente porque as dimensões da sustentabilidade possibilitam a observação sistêmica do atendimento satisfatório das dimensões envolvidas nas decisões e ações territoriais. Portanto, seja com enfoque ambientalista, seja ecológico, essas abordagens econômicas propõem a visão sistêmica da sustentabilidade.

A existência de correntes econômicas da sustentabilidade vem confirmar a diversidade interpretativa sobre um assunto de extrema importância e complexidade. A principal lição que ambas as correntes trazem sobre as questões do desenvolvimento sustentável é a ênfase na necessidade de respeitar o limite de 
suporte do planeta, apesar das diferentes posições evidenciadas pelas correntes acerca do debate sobre o desenvolvimento sem crescimento (DALY, I99I) ou crescimento sem destruição (SACHS, 2002).

\subsection{AS DIMENSÕES E OS CRITÉRIOS DA SUSTENTABILIDADE}

A compreensão e a aplicação das dimensões e dos critérios da sustentabilidade aos negócios tendem a garantir que os gestores tomem decisões sustentáveis nas organizações. Entretanto, conforme Ciegis, Ramanauskiene e Martinkus (2009), como os novos conhecimentos e as experiências afetam a compreensão dos problemas e a busca por soluções, sustentabilidade é um conceito em constante mudança, e essas são as razões para estudar em profundidade suas dimensões.

No Relatório Brundtland, são destacados três componentes fundamentais para o desenvolvimento sustentável: proteção ambiental, crescimento econômico e equidade social (COMISSÃO MUNDIAL SOBRE MEIO AMBIENTE E DESENVOLVIMENTO, I988). Esses três componentes representam as dimensões da sustentabilidade para muitos autores. Para Ciegis, Ramanauskiene e Martinkus (2009, p. 30), "desenvolvimento sustentável não se trata de uma escolha entre a proteção ambiental e o progresso social, mas de um esforço maior para desenvolvimento econômico e social que seja compatível com a proteção ambiental". Portanto, de forma geral, o desenvolvimento sustentável envolve três aproximações fundamentais, que são inter-relacionadas e complementares: econômica, social e ambiental, que representam as dimensões da sustentabilidade (CIEGIS; RAMANAUSKIENE; MARTINKUS, 2009).

Desses três componentes fundamentais, surge o que Elkington (I999) denominou como triple bottom line, ou seja, são os três pilares norteadores de decisões e ações relacionadas à gestão organizacional, aproximando o conceito de responsabilidade social corporativa do conceito da sustentabilidade.

Assim, uma organização pode criar valor ou destruí-lo, de acordo com seu desempenho, fundamentado, simultaneamente, no pilar econômico, social e ambiental (ELKINGTON, I999). Conforme Dias (2008), o mais importante, na abordagem das três dimensões da sustentabilidade empresarial, é o equilíbrio dinâmico entre as dimensões econômica, social e ambiental.

$\mathrm{Na}$ perspectiva de Elkington (2004), tal equilíbrio poderá ser alcançado quando a organização considerar a integração dos três pilares da sustentabilidade, ao observar o que ele chama de 3 "Ps" (em português, PPL). Os 3 "Ps" são compostos por "pessoa" (people), que aborda o capital humano de uma sociedade ou organização; "planeta” (planet), relacionado com o capital natural da socie- 
dade ou da organização; e "lucro" (profit), que trata dos resultados econômicos positivos da organização.

O triple bottom line é uma importante ferramenta de apoio à tomada de decisões para gestores organizacionais. Como afirma Elkington (I999, 2004), mais de cem definições de sustentabilidade podem ser encontradas, e a transição do capitalismo sustentável será uma das mais complexas que a humanidade terá de negociar.

Ciegis, Ramanauskiene e Martinkus (2009) apresentam uma classificação em categorias de debate sobre a sustentabilidade, divididas em: conceitual, que foca a origem etimológica do termo; contextual, que discute a posição institucional relacionada aos consensos internacionais; acadêmica, que inclui propostas teóricas, conceituais e metodológicas; e, finalmente, a categoria geopolítica, que discute a distinção do conceito de desenvolvimento entre os países desenvolvidos e subdesenvolvidos.

Uma das abordagens mais importantes sobre as dimensões da sustentabilidade nos últimos anos é a de Ignacy Sachs (2002), com suas oito dimensões da sustentabilidade, apresentadas a seguir: a dimensão social propõe homogeneidade social, distribuição de renda justa, qualidade de vida e igualdade social; a cultural sugere equilíbrio, tradição e inovação, autonomia na elaboração de projetos nacionais integrados e a combinação entre confiança e abertura para o mundo; a ecológica propõe a preservação do capital natural e a limitação no uso desses recursos; a ambiental engloba o respeito aos ecossistemas naturais; a territorial trata do equilíbrio entre as configurações urbanas e rurais, da melhoria do ambiente urbano e das estratégias de desenvolvimento de regiões; a econômica aborda o equilíbrio econômico entre setores, a segurança alimentar, a modernização dos meios produtivos, a realização de pesquisas científicas e tecnológicas e a inserção na economia internacional; a dimensão política nacional envolve a democracia, os direitos humanos e a implantação de projetos nacionais em parceria com os empreendedores; por fim, a dimensão política internacional trata da promoção da paz e da cooperação internacional, do controle financeiro internacional, da gestão da diversidade natural e cultural e da cooperação científica e tecnológica.

As oito dimensões da sustentabilidade propostas por Sachs (2002) permitem visualizar os elementos envolvidos e afetados pelas interações existentes em um contexto de desenvolvimento territorial ou regional, mas, na perspectiva organizacional, os critérios abordados pelas dimensões não são claros. Como exemplo, destaca-se o critério que trata da distribuição de renda justa, encontrado dentro da dimensão social, mas que poderia estar contemplado não somente, mas principalmente, na dimensão econômica, ou, ainda, o critério da segurança alimentar, que se encontra dentro da dimensão econômica, mas que poderia compor, principalmente, a dimensão social e/ou política. 
As diferentes compreensões desses critérios ocorrem em razão das interpretações que envolvem os estudos da sustentabilidade, não sendo, portanto, uma questão de definir o certo e o errado.

Diante do exposto, entre as principais dimensões da sustentabilidade, três delas são consensuais: econômica, social e ambiental. Contudo, tal consenso não é identificado em relação aos critérios que compõem cada uma das dimensões, por serem provenientes de visões de mundo individuais dos pesquisadores. A falta de clareza na definição dos critérios mais confunde do que ajuda, ao se aplicarem as dimensões da sustentabilidade nas tomadas de decisões organizacionais.

É importante esclarecer que se consideram como critério neste artigo os elementos que compõem ou caracterizam cada uma das dimensões, ou seja, são partes menores ou específicas que constituem as dimensões da sustentabilidade.

Conforme Hinterberger et al. (I997 apud CIEGIS; RAMANAUSKIENE; MARTINKUS, 2009), quatro níveis de aplicação das dimensões da sustentabilidade são identificados: o primeiro é o micronível, que inclui empresas e consumidores; o segundo é o mesonível, incluindo aspectos fiscais, monetários e de distribuição; e o metanível, que inclui os objetivos sociais.

Neste estudo, optou-se pelo uso das três dimensões básicas da sustentabilidade (triple bottom line) e pela definição específica, para este artigo, dos critérios que compõem cada uma das dimensões, de acordo com o micronível de aplicação, ou seja, o nível de empresas (organizacional).

A abordagem sistêmica das dimensões da sustentabilidade é uma alternativa viável de compreensão das conexões existentes entre dimensões e seus critérios (componentes ou elementos). Com a definição dos critérios que compõem cada uma das dimensões da sustentabilidade, é possível mapear as conexões existentes entre os critérios e, consequentemente, compreender as influências de determinado(s) critério(s) sobre certa(s) dimensão(ões).

Se as decisões tomadas pelas organizações estiverem alinhadas a esses critérios e considerarem simultaneamente as dimensões, existe grande possibilidade de alcance da sustentabilidade.

O uso das dimensões da sustentabilidade e a identificação das relações existentes entre os critérios permitem superar as tradicionais decisões limitadas, pois se exige pensamento sistêmico, contribuindo para o alcance da sustentabilidade por meio do equilíbrio entre (e intra) dimensões. Supondo o comprometimento, a responsabilidade e a consciência dos envolvidos, o consenso sobre o que é sustentável ou insustentável deve partir da interação entre decisores dentro da organização, entre outros envolvidos e afetados, ou seja, é um processo interativo contínuo. 


\subsection{DECISÕES ORGANIZACIONAIS}

Desde os tempos mais remotos da existência da humanidade, as decisões sempre estiveram presentes na busca pela sobrevivência e pela evolução da humanidade. Ainda que de forma inconsciente ou irracional, as decisões tomadas pelo homem, ao longo dos tempos, influenciaram indivíduos, civilizações, sociedades, economias e organizações. Com a formação das sociedades e sua dependência das organizações, as decisões tomadas dentro das empresas passaram a influenciar o desenvolvimento econômico de muitas regiões e nações. As opções ou alternativas envolvidas nas decisões são obtidas por meio de experiências, informações e interesses que levam o decisor a determinadas escolhas.

A palavra decisão é formada pelos termos latinos de (que significa parar, extrair, interromper) e caedere (que significa cindir, cortar), que, originalmente, significa "parar de cortar" ou "deixar fluir", e sua definição está relacionada à escolha da melhor alternativa de solução entre as possíveis (GOMES; GOMES; ALMEIDA, 2006).

Sob a influência do pensamento filosófico positivista e do liberalismo econômico, a teoria da administração científica de Taylor passa a enfocar a eficiência da empresa, sustentando-se na extrema racionalidade e no conceito de homem econômico. Esse conceito considera o homem um ser eminentemente racional e que, ao tomar uma decisão, conhece todos os cursos de ação disponíveis, apesar de considerar que o comportamento humano é simples e previsível (MOTTA; VASCONCELOS, 2002).

Conforme Shimizu (200I), o homem economista (homem econômico) é aquele que trabalha com toda a confusão e complexidade do "mundo real" e que, ao escolher a melhor alternativa, adota um "padrão ótimo" da realidade. Portanto, as decisões que se fundamentam no conceito de homem econômico buscam encontrar a alternativa ótima entre todas as opções.

Ao abordar o comportamento administrativo nas organizações, Herbert Alexander Simon (I97I) desenvolveu o estudo da teoria das decisões organizacionais. As relevantes contribuições de Simon ao estudo precursor da tomada de decisões racionais limitadas nas organizações lhe renderam, em I978, o Prêmio Nobel de Economia. Em seu discurso, ao receber o Prêmio Nobel, "Simon reafirma [...] a sua crença na necessidade de adotar uma visão mais realista do comportamento humano, muitas vezes, incerto e imprevisto, influenciado por conflitos e interesses pessoais específicos dos indivíduos" (MOTTA; VASCONCELOS, 2002, p. 106).

A fronteira entre racionalidade e irracionalidade seria o ponto central do debate em relação ao comportamento humano nas organizações e aos reflexos deste nas decisões. Em seu livro, Simon (I97I) explica que, diferentemente do 
conceito de homem econômico que procura lidar com o mundo real em toda a sua complexidade, o homem administrativo considera apenas os fatores mais relevantes e fundamentais, pois reconhece que sua percepção de mundo é apenas uma drástica simplificação do mundo real.

Assim, a teoria das decisões surge como uma tentativa de superação dos postulados da teoria econômica tradicional, representada pelo conceito de "homem econômico", defendido por Frederick Winslow Taylor, por meio do conceito de "homem administrativo" proposto por Herbert Simon (I97I), que vê o processo decisório como sinônimo de processo administrativo.

A teoria da decisão não é uma teoria descritiva ou explicativa, já que não faz parte de seus objetivos descrever e/ou explicar por que as pessoas (ou instituições) agem de determinada forma ou tomam certas decisões (GOMES; GOMES; ALMEIDA, 2006, p. 2I). Segundo Simon (I955), a tarefa da tomada de decisão racional é selecionar a alternativa que resulta de um conjunto de todas as possíveis e mais desejáveis consequências.

De acordo com Miller, Hickson e Wilson (2004), Herbert Simon argumenta que os tomadores de decisões são incapazes de operar em condições de racionalidade perfeita, em razão de suas próprias limitações cognitivas; da complexidade das organizações modernas; da falta de clareza das questões a serem decididas; da diversidade de interpretações, além da possibilidade de informações incompletas ou inexistentes; da incerteza na definição de critérios de avaliação de escolhas; e, finalmente, da limitação de tempo e energia disponíveis para a tomada de decisão.

Além disso, existem os gargalos na tomada de decisão que emperram o processo decisório nas organizações e que, segundo Rogers e Blenko (2008, p. I), "podem ocorrer sempre que exista ambigüidade ou estresse com relação a quem pode decidir". Tais limitações, somadas às fraquezas humanas e aos fatores internos e externos às organizações que também reduziriam o grau de racionalidade, fariam com que os resultados das decisões tomadas fossem apenas "satisfatórios”, e não ótimos (CHECKLAND, 2000; MILLER; HICKSON; WILSON, 2004).

Assim, "Simon aceita que os administradores realizem operações dentro do conceito de uma 'racionalidade limitada'" (MILLER; HICKSON; WILSON, 2004, p. 284), resultando em algo satisfatório. Portanto, o modelo racional de tomada de decisão proposto por Simon (I97I) vem contribuindo para o alcance de objetivos essencialmente econômicos das organizações e para a consequente expansão do modo capitalista de produção.

A racionalidade implícita no processo decisório é relevante porque permite a organização de ideias, a identificação de alternativas e a definição de uma linha de ação organizacional. Além disso, a teoria das decisões de Herbert Simon também é um importante marco para a abordagem sistêmica das organizações por fun- 
damentar a visão hard do pensamento sistêmico, "[...] que permeia tanto a teoria ortodoxa das organizações como a ciência da gestão simoniana" (CHECKLAND, 2000, p. 49).

Ao abordar o pensamento sistêmico, Checkland (2000) faz a distinção entre pensamento sistêmico hard, que busca obter resultados ótimos ou satisfatórios, por meio de modelos que simplificam a realidade, e o pensamento sistêmico soft, que usa a visão de mundo dos indivíduos na estruturação de problemas complexos e na aprendizagem organizacional, com a adoção de metodologias sistêmicas.

O pensamento sistêmico hard tem como premissa a clara definição de problemas na estruturação do processo de tomada de decisões, pois sustenta a visão objetiva e racional do mundo sistêmico.

De acordo com Donaires (2008, p. 3), "o pensamento sistêmico hard propõe que os sistemas sociais existem na prática e determinam o comportamento dos atores participantes do sistema".

Segundo Rodrigues (2006, p. 82), "a visão de mundo - hard - acredita que todo problema pode ser definido e, portanto, a sua respectiva solução poderá ser encontrada”. Logo, o pensamento sistêmico hard é uma forma de ver o mundo (a realidade) em que o decisor ou pesquisador, ao pensar em fenômenos, processos, situações ou variáveis, utiliza métodos quantitativos, objetivos e deterministas na estruturação de seus conhecimentos e na busca por explicações e respostas.

Entretanto, esse enfoque extremamente racional, determinista e fragmentário, presente nos processos decisórios funcionalistas, desconsidera a relação, o efeito e as consequências dentro de um conjunto de fatores, variáveis ou dimensões envolvidos. O processo decisório investigado cientificamente por Simon é uma forma de simplificação do que é complexo, seguindo o pensamento de Bachelard (MORIN, 2008).

Mackness (2006, p. 87) argumenta que "a abordagem defendida por Simon para solucionar problemas de uma forma científica é simples e não-sustentável”. Para ele,

[...] essa abordagem não é sustentável porque a wickeness (perniciosidade, severidade) da situação problemática não nos permite "estabelecer metas" ou estarmos certos sobre "as diferenças entre a situação presente e a meta" (MACKNESS, 2006, p. 87).

A abordagem das decisões organizacionais pelo enfoque de sua complexidade é uma tentativa de superar o funcionalismo presente no conceito de homem econômico e administrativo. A complexidade que envolve as decisões organiza- 
cionais pode ser vista de formas diferentes, podendo estar relacionada ao elevado número de variáveis com que o decisor lida (GOMES; GOMES; ALMEIDA, 2006), à dificuldade de definir o problema (CHECKLAND, 2000, MILLER; HICKSON; WILSON, 2004) ou, ainda, à ideia de que não existe o simples, pois tudo é complexo (MORIN, 2008).

Para Gomes, Gomes e Almeida (2006), as decisões complexas se caracterizam pelo elevado grau de dificuldade que o indivíduo ou os grupos de indivíduos enfrentam, em razão da necessidade de atender a múltiplos objetivos, e seus impactos frequentemente são difíceis de ser adequadamente identificados. Já Checkland (2000) defende que a maior causa da complexidade são as diferentes visões de mundo. Estas visões de mundo elevam a complexidade das decisões, fazendo com que a própria identificação do problema a ser resolvido se torne uma tarefa de elevada dificuldade (MACKNESS, 2006).

Em relação à complexidade presente na tomada de decisão, Miller, Hickson e Wilson (2004, p. 285) explicam que, nesses casos, "as informações necessárias poderão ser dificilmente coletadas como categorizadas; soluções potenciais podem ser de difícil reconhecimento e sua implementação pode criar novos problemas".

De acordo com Miller, Hickson e Wilson (2004, p. 299), "decisões podem variar em conteúdo [sobre o que versam] e em processo [como a decisão caminha no interior da organização] e podem variar em importância [operacionais ou estratégicas]". Portanto, nas situações em que os problemas são de extrema complexidade, a tomada de decisão racional limitada estará comprometida, mesmo com o pensamento sistêmico hard (hard systems thinking), que também pressupõe a existência de um problema visivelmente estruturado.

Nessas situações de extrema complexidade, as decisões satisfatórias, presentes na teoria das decisões de Simon, são desejadas, mas a simplificação ou fragmentação não.

Proveniente das limitações do pensamento sistêmico hard em lidar com a complexidade do ambiente social e organizacional, o pensamento sistêmico soft, originalmente desenvolvido na década de I960 por Peter Checkland e seu grupo de pesquisadores, reconhece o elevado nível de complexidade, subjetividade e interpretação envolvido nas situações cotidianas e utiliza métodos qualitativos na tentativa de compreender as situações sociais e organizacionais e aprender com elas.

O pensamento sistêmico soft se sustenta principalmente no paradigma interpretativista, em que a realidade é interpretada e construída pelo pesquisador ou decisor, a partir de interesses, experiências e compreensões individuais. Essa forma de pensamento sistêmico reconhece a existência de certa problemática, de difícil identificação. Nesse caso, não tem por objetivo "encontrar uma solução, mas buscar aprender sobre a realidade e orientar ações sobre ela" (RODRIGUES, 
2006, p. 82). Isso, possivelmente, deve-se ao fato de que o ser humano está rodeado de sistemas altamente complexos que influenciam, cada vez mais, muitos aspectos de sua vida (CAPRA, 2005).

Diante do perfil subjetivo do pensamento sistêmico soft, "a idéia de 'sistema' emerge como uma estrutura conceitual robusta para facilitar a investigação de situações e problemas reais complexos, a fim de melhor compreendê-los e resolvê-los" (FREITAS; COTA JUNIOR; CHENG, 2006, p. 3). Entre as principais metodologias sistêmicas soft, Schwaninger (2006) destaca o Interactive Planning (de Ackoff), a Soft Systems Methodology (de Checkland) e a Critical Systems Heuristics (de Ulrich).

Não é o objetivo aqui, contudo, abordar cada uma das metodologias, mas, de forma geral, considera-se que todas elas propõem a compreensão da problemática das situações na busca por soluções, gerando um processo de aprendizagem.

\section{CONTRIBUIÇÃO METODOLÓGICA AO ESTUDO DOS NÍVEIS DE COMPLEXIDADE DAS DECISÕES E DA SUSTENTABILIDADE}

\subsection{DEFINIÇÃO DOS NÍVEIS DE COMPLEXIDADE DAS DECISÕES}

Este artigo é um ensaio teórico que propõe a compreensão da sustentabilidade por meio da classificação dos níveis de decisões relevantes para as organizações. Com o objetivo de estudar as decisões e suas influências na sustentabilidade, elas foram classificadas em três níveis de complexidade: decisões simples, medianas e complexas.

Há um consenso no estudo das decisões em relação à existência de decisões simples e complexas (GOMES; GOMES; ALMEIDA, 2006). Além disso, conforme a hierarquia de sistemas de Boulding (1966), as organizações estão entre os sistemas mais complexos, porém, para Miller, Hickson e Wilson (2004), muitas decisões e pesquisas organizacionais estão sustentadas nos sistemas mais simples. Essa visão das organizações como sistemas simples pode ter dois motivos: o primeiro é que o indivíduo se utiliza da simplicidade, porque é incapaz de lidar com a complexidade; a segunda possibilidade é de que o indivíduo tem condições de lidar com um nível maior de complexidade, entretanto prefere a simplicidade, como sugere Morin (2008).

Em razão desses dois motivos, sugeriu-se a inclusão de um nível decisório mediano como forma de enquadrar os pesquisadores e decisores que se utili- 
zam do conceito de homem administrativo de Simon (I97I) ou o pensamento sistêmico hard. As decisões medianas são aquelas que indicam uma transição das decisões simples para as complexas ou, ainda, da complexa para a simples. Um decisor que sempre tomou decisões simples tende a ter dificuldades de tomar decisões complexas de um momento para o outro, em decorrência da forma como vê os outros indivíduos e o mundo em que vive, além de sua possível insegurança e despreparo para lidar com múltiplas variáveis simultaneamente ou estruturar situações-problema desestruturadas.

\subsection{DEFINIÇÃO DE CRITÉRIOS DAS DIMENSÕES DA SUSTENTABILIDADE E O MAPEAMENTO DE SUAS RELAÇÕES}

As dimensões da sustentabilidade são compostas por elementos menores que as caracterizam. Entretanto, como já discutido na seção das dimensões da sustentabilidade, constatou-se que os critérios dessas dimensões não atendem às necessidades de aplicação e análise organizacional. Por esse motivo, optou-se pela escolha de critérios especificamente direcionados às organizações na composição de cada uma das três dimensões da sustentabilidade. As dimensões utilizadas na matriz foram caracterizadas por critérios que atendem aos aspectos de viabilidade econômica, responsabilidade social e consciência ambiental das decisões relevantes tomadas na organização.

Dessa forma, os critérios que compõem a dimensão econômica, propostos neste artigo, estão relacionados às finanças organizacionais. Segundo Gitman (2002), as finanças organizacionais envolvem a análise e o planejamento financeiro, bem como as decisões de investimento e financiamento.

Entre os critérios da dimensão econômica estão: equilíbrio entre recebimentos e pagamentos no fluxo de caixa, inclusive preços e salários justos; adequado investimento em estrutura, equipamento, treinamento e marketing; e financiamento viável de curto, médio e longo prazos.

Os critérios escolhidos para compor a dimensão social estão relacionados ao bem-estar dos envolvidos com a organização (stakeholders), considerando a perspectiva da responsabilidade social. Conforme Martinelli (I997), a empresa, como organização social, envolve, em uma relação de interdependência, os interesses de stakeholders como clientes, funcionários, fornecedores, comunidade e acionistas. Assim, entre os critérios da dimensão social a serem atendidos pela organização, destacam-se: segurança; bem-estar e satisfação dos clientes e dos colaboradores; parceria com os fornecedores; interação com o governo; apoio às organizações não governamentais (ONGs); e envolvimento e participação em projetos sociais e comunitários locais. 
Em relação à dimensão ambiental, Elkington (2004) destaca que as empresas tendem a gerenciar o ciclo de vida de seus produtos, desde a extração até a reciclagem e a eliminação. Com base nessa tendência, foram considerados como critérios da dimensão ambiental: análise, prevenção e redução contínua de emissão de resíduos tóxicos; processos e tecnologias de produção mais limpa; o ciclo de vida dos produtos no caso das indústrias; participação em projetos de proteção, preservação e conservação da fauna e flora local; e incentivo aos projetos de educação ambiental e consciência ecológica.

Definidos os critérios das dimensões da sustentabilidade, buscou-se identificar as relações (sistêmicas) existentes entre cada um desses critérios. Com o mapeamento das relações existentes entre cada um dos critérios das dimensões, torna-se possível saber como uma dimensão tende a influenciar a outra, sem a necessidade de fazer abordagens e suposições dimensionais genéricas e descomprometidas.

Dessa forma, apresenta-se, na Tabela I, o mapeamento das relações entre os critérios que compõem as dimensões da sustentabilidade.

\section{TABELA I}

MAPEAMENTO DAS RELAÇÕES ENTRE OS CRITÉRIOS DAS DIMENSÕES DA SUSTENTABILIDADE

\begin{tabular}{|c|c|c|c|c|c|}
\hline \multirow{2}{*}{$\begin{array}{l}\text { DIMENSÕES } \\
\text { DA } \\
\text { SUSTENTABILIDADE }\end{array}$} & \multirow{2}{*}{$\mathrm{N}^{\circ}$} & \multirow{2}{*}{$\begin{array}{l}\text { CRITÉRIOS DAS } \\
\text { DIMENSÕES }\end{array}$} & \multicolumn{3}{|c|}{ DIMENSÕES DA SUSTENTABILIDADE } \\
\hline & & & ECONÔMICA & SOCIAL & AMBIENTAL \\
\hline \multirow{3}{*}{ Econômica } & 1 & Fluxo de caixa & 2,3 & $4,5,6,7,8,9$ & $10,11,12,13,14$ \\
\hline & 2 & Investimentos & 1,3 & $4,5,6,7,8,9$ & $10,11,12,13,14$ \\
\hline & 3 & Financiamento & 1,2 & $4,5,6,7,8,9$ & $10,11,12,13,14$ \\
\hline \multirow{6}{*}{ Social } & 4 & Clientes & $1,2,3$ & $5,6,7,9$ & $10,11,12,13,14$ \\
\hline & 5 & Colaboradores & $1,2,3$ & $4,6,7,9$ & $10,11,12,13,14$ \\
\hline & 6 & Fornecedores & $1,2,3$ & $4,5,7,9$ & $10,11,12,13,14$ \\
\hline & 7 & Governos & $1,2,3$ & $4,5,6,8,9$ & $10,13,14$ \\
\hline & 8 & ONGs & $1,2,3$ & 7,9 & $10,13,14$ \\
\hline & 9 & Comunidade local & $1,2,3$ & $4,5,6,7,8$ & $10,11,12,13,14$ \\
\hline
\end{tabular}




\section{TABELA I (CONCLUSÃo)}

\section{MAPEAMENTO DAS RELAÇÕES ENTRE OS CRITÉRIOS DAS DIMENSÕES DA SUSTENTABILIDADE}

\begin{tabular}{llllrl}
\hline \multirow{2}{*}{$\begin{array}{l}\text { DIMENSÕES } \\
\text { DA } \\
\text { SUSTENTABILIDADE }\end{array}$} & $\begin{array}{l}\text { No } \\
\text { CRITÉRIOS DAS } \\
\text { DIMENSÕES }\end{array}$ & \multicolumn{3}{c}{ DIMENSÕES DA SUSTENTABILIDADE } \\
\cline { 2 - 5 } & 10 & Resíduos & $1,2,3$ & $4,5,6,7,8,9$ & $11,12,13,14$ \\
\cline { 2 - 5 } & 11 & Processos & $1,2,3$ & $4,5,6,9$ & $10,12,13,14$ \\
\cline { 2 - 5 } Ambiental & 12 & Produtos & $1,2,3$ & $4,5,6,9$ & $10,11,13,14$ \\
\cline { 2 - 5 } & 13 & Fauna e flora & $1,2,3$ & $4,5,6,7,8,9$ & $10,11,12,14$ \\
\cline { 2 - 5 } & 14 & Educ. ambiental & $1,2,3$ & $4,5,6,7,8,9$ & $10,11,12,13$ \\
\hline
\end{tabular}

Fonte: Adaptada de Maia (20I0).

As relações existentes entre os critérios das três dimensões da sustentabilidade são identificadas de acordo com as numerações vinculadas à esquerda dos critérios. As enumerações têm como objetivo facilitar a visualização das interações, reduzindo o tamanho da tabela para a rápida consulta, compreensão e apoio às decisões. Dessas interações apresentadas, não significa que as decisões tomadas estarão diretamente envolvidas com todos os critérios das dimensões, mas, naquelas decisões em que se considera certo o critério das dimensões, a influência nos demais critérios deverá ser verificada, e a Tabela i facilita esse processo.

Para fins de exemplificação, utilizar-se-á uma situação em que a empresa (e seu decisor) precisa avaliar a decisão de compra de um novo equipamento a ser usado em sua linha produtiva. Dentro da dimensão econômica, considera-se que a decisão de compra do equipamento está classificada no critério de investimento (2), que, de acordo com a Tabela I, deverá ser avaliado em termos de critério do equilíbrio de fluxo de caixa (I) e do critério relacionado à necessidade de futuro financiamento (3).

Em relação à dimensão social, esse critério de investimento (2) relaciona-se ao critério de satisfação dos clientes (4), no caso de haver aumento das vendas, aumento da produção, geração de empregos e atendimento satisfatório do critério relacionado aos colaboradores (5), enquanto se promovem parcerias com os fornecedores (6), gerando maior recolhimento de impostos cobrados pelo critério relacionado ao governo (7). No caso desse investimento em específico, o critério ONGs (8) talvez não necessite ser considerado, desde que o investimento 
não perturbe a comunidade ou o meio ambiente, mas o critério de comunidade local (9) pode ser atendido com o aumento de empregos diretos e indiretos.

$\mathrm{Na}$ dimensão social, o investimento em um equipamento pode tanto reduzir como aumentar os resíduos (critério Io) como exigir, dentro do critério II, uma readequação dos processos para uma produção mais limpa e, com o critério (I2), dos produtos atendidos ou não, em termos de qualidade e ciclo de vida.

Os investimentos tendem a gerar recursos futuros que podem ser aplicados no critério (I3), que envolve a preservação e conservação da fauna e flora da região, ou no critério (I4), de educação ambiental. Como se pode perceber, algumas suposições foram feitas apenas para exemplificar a aplicação da Tabela I, sendo possível constatar que, nesse caso, a empresa tomaria uma decisão que contemplaria, de forma satisfatória, todos os critérios e as dimensões da sustentabilidade, contribuindo para o alcance da sustentabilidade.

$\mathrm{O}$ atendimento satisfatório e simultâneo das dimensões da sustentabilidade classifica a decisão em seu nível mais alto, o nível complexo. Assim, para os decisores que procuram elevar o nível de complexidade de suas decisões, o mapeamento das interações se torna essencial.

O pensamento sistêmico soft é evidenciado no mapeamento apresentado na Tabela I e na matriz apresentada no Quadro I, pois, apesar de definidos especificamente para o presente estudo, tanto as dimensões e seus critérios quanto as interações entre os critérios são provenientes de uma interpretação dos autores e podem ser revistos e reestruturados conforme as visões de mundo ou interpretações do pesquisador ou decisor que venha a utilizar a tabela, sem esquecer o rigor científico.

\subsection{A ESTRUTURA DA MATRIZ DOS NÍVEIS DE COMPLEXIDADE DAS DECISÕES ORGANIZACIONAIS DIRECIONADAS À SUSTENTABILIDADE}

Do cruzamento entre os três níveis de complexidade das decisões e as três dimensões da sustentabilidade, foi possível estruturar a matriz dos níveis de complexidade das decisões organizacionais direcionadas à sustentabilidade.

Essa matriz é uma estrutura qualitativa de classificação das decisões, e com ela é possível identificar em qual nível de decisão se classificam as decisões relevantes tomadas por um decisor, a partir da identificação de quantos e quais critérios e dimensões da sustentabilidade foram atendidos pela organização.

Essa matriz foi desenvolvida justamente para o uso em organizações de diversos ramos de atividades e setores produtivos ou ainda em pesquisas organizacionais. Portanto, a estrutura da matriz dos níveis de complexidade das deci- 
sões direcionadas à sustentabilidade (Quadro I) está sustentada nos critérios das dimensões da sustentabilidade e em suas interações (Tabela I).

A estrutura da matriz com as dimensões da sustentabilidade e os níveis de complexidade das decisões são apresentados no Quadro I.

\section{QUADRO I}

\section{MATRIZ DOS NÍVEIS DE COMPLEXIDADE DAS DECISÕES}

DIRECIONADAS A SUSTENTABILIDADE

\begin{tabular}{|c|c|c|c|}
\hline \multicolumn{4}{|c|}{$\begin{array}{l}\text { MATRIZ DOS NIVEEIS DE COMPLEXIDADE DAS DECISÕES } \\
\text { ORGANIZACIONAIS DIRECIONADAS À SUSTENTABILIDADE }\end{array}$} \\
\hline \multirow{2}{*}{$\begin{array}{l}\text { DIMENSÕES DA } \\
\text { SUSTENTABILIDADE }\end{array}$} & \multicolumn{3}{|c|}{ NIVEIS DE COMPLEXIDADE DAS DECISÕES } \\
\hline & SIMPLES & MEDIANAS & COMPLEXAS \\
\hline \multirow{3}{*}{ Econômica } & - Fluxo de caixa & - Fluxo de caixa & - Fluxo de caixa \\
\hline & - Investimento & - Investimento & - Investimento \\
\hline & - Financiamento & - Financiamento & - Financiamento \\
\hline \multirow{6}{*}{ Social } & \multirow{6}{*}{-} & - Clientes & - Cliente \\
\hline & & - Colaboradores & - Colaboradores \\
\hline & & - Fornecedores & - Fornecedores \\
\hline & & - Governos & - Governo \\
\hline & & - ONGs & - ONGs \\
\hline & & - Comunidade local & - Comunidade local \\
\hline \multirow{5}{*}{ Ambiental } & \multirow{5}{*}{-} & \multirow{5}{*}{-} & - Resíduos \\
\hline & & & - Processos \\
\hline & & & - Produtos \\
\hline & & & - Fauna e flora \\
\hline & & & - Educação ambiental \\
\hline
\end{tabular}

Fonte: Adaptado de Maia (20Iо).

No que se refere aos níveis de decisões, pode-se observar no Quadro I que as decisões simples buscam atender de forma ótima a apenas uma dimensão, a 
econômica. É nesse nível de decisão que se encontra grande parte das decisões organizacionais que atuam em diversos setores. Em razão de seu enfoque direcionado apenas à dimensão econômica, o nível de decisão simples tem influenciado negativamente outras dimensões da sustentabilidade, gerando sérios problemas, principalmente ambientais e sociais.

O nível mediano de decisões sugere primeiramente o atendimento da dimensão econômica, tendo a possibilidade, quando conveniente para a empresa, de atender à dimensão social (como ilustrado no Quadro I) ou ambiental. O nível mediano pode representar uma tentativa de elevar o nível de complexidade das decisões de certo indivíduo ou certa organização. Ainda assim, esse nível de decisão tende a gerar influências insatisfatórias à sustentabilidade, por desencadear ações sequenciais (soma de partes) e não simultâneas e, ainda, por desconsiderar as outras dimensões de seu processo decisório.

O último nível decisório é reconhecido como complexo por caracterizar as decisões que procuram atender às três principais dimensões da sustentabilidade simultaneamente, gerando influências sistêmicas satisfatórias nos critérios e, consequentemente, na sustentabilidade. Nesse nível decisório, o profissional precisa ter elevada competência e pensamento sistêmico desenvolvido, saber trabalhar de forma participativa e estar aberto à aprendizagem contínua, diante das situações enfrentadas, para influenciar satisfatoriamente, com suas complexas e relevantes decisões, a sustentabilidade. O nível complexo de decisões é aquele em que os decisores procuram, de forma sistêmica, tomar decisões que atendam simultaneamente às dimensões econômica, social e ambiental, entre outras, quando for o caso.

\section{CONSIDERAÇÕES FINAIS}

Dos excessos do sistema capitalista de produção e das discussões, em nível global, de seus efeitos negativos na sociedade e no ambiente natural nas últimas décadas, emerge o conceito de desenvolvimento sustentável. No centro dessas discussões, estão as organizações e as decisões tomadas por seus gestores na busca desenfreada pelo crescimento econômico, em detrimento dos aspectos sociais e dos fatores naturais. Com o crescente reconhecimento do conceito de sustentabilidade pelas organizações, as decisões organizacionais exigem uma abordagem mais complexa que envolva, simultaneamente, três dimensões da sustentabilidade, entre elas: econômica, social e ambiental.

Entretanto, apesar do consenso em relação às três dimensões da sustentabilidade, o mesmo não ocorre quando se trata dos critérios que compõem cada 
uma das dimensões. Diante da complexidade das decisões organizacionais, ao abordar as três dimensões simultaneamente, e da subjetividade na definição dos critérios que compõem a dimensão da sustentabilidade, este artigo propôs uma estrutura denominada matriz de complexidade dos níveis de decisões direcionadas à sustentabilidade.

Composta pelo cruzamento entre os três níveis de decisões (simples, medianas e complexas) e as três dimensões da sustentabilidade, essa matriz permite ao decisor identificar o nível de complexidade de suas decisões, classificando-as com base no atendimento simultâneo das dimensões da sustentabilidade.

Assim, de acordo com a matriz, considera-se que as decisões simples tomadas nas organizações enfocam a dimensão econômica, mas desconsideram as dimensões social e ambiental. Já as decisões medianas, ainda que considerem sequencialmente (e não simultaneamente) a dimensão econômica e social (ou ambiental), desconsideram a dimensão ambiental (ou social). Contudo, as decisões organizacionais complexas, por atenderem simultaneamente às três dimensões da sustentabilidade, tendem a contribuir para o alcance da sustentabilidade.

Salienta-se, ainda, que a matriz é uma estrutura flexível e permite ajustes a adequações necessárias, de acordo com a atividade ou o setor de atuação da organização, sendo possível, inclusive, considerar outros critérios e dimensões relevantes.

\section{AN UNDERSTANDING OF SUSTAINABILITY THROUGH THE LEVELS OF ORGANIZATIONAL COMPLEXITY OF DECISIONS}

\section{ABSTRACT}

In a capitalist society, organizational decisions, usually aimed at the economic dimension, triggered or exacerbated the socio-environmental problems in many countries. In the search for solutions to such problems arises in the I980s the concept of sustainable development proposing to meet the needs of present generations without compromising the care of the needs of future generations (COMISSÃO MUNDIAL SOBRE MEIO AMBIENTE E DESENVOLVIMENTO, I988). Considered a subjective concept and open to interpretation, sustainable development, includes support of three essential dimensions: the economic, the social and the environmental one. Thus, under the organizational perspective, to make decisions that contribute to sustainability, has become at least a big challenge, because beyond the economic dimension, decision makers must 
deal simultaneously with the social and environmental dimensions. This shows that there are levels of decisions defined as simple, average, and complex and that these levels tend to influence or compromise the achievement of sustainability differently, depending on the service satisfactory or not of the dimensions. Faced with this challenge this paper, while theoretical essay aims to present an understanding of sustainability through the level of complexity of organizational decisions. To achieve this goal, it is proposed the relevant criteria that compose each dimension of sustainability, identifying the relationships (systemic) that may occur between the criteria for organizational decision making. As a result of crossing between levels of complexity of decisions and the dimensions of sustainability, a structure of a complex matrix of decisions was obtained to address sustainability. The qualitative matrix shows how the decision-maker or researcher may classify the decisions considered relevant and how he can make complex decisions that integrate satisfactorily the three dimensions of sustainability.

\section{KEYWORDS}

Sustainability; Dimensions of sustainability; Dimensions of sustainability criteria; Levels of decisions; Organizations.

\section{UN ACUERDO DE SOSTENIBILIDAD A TRAVÉS DEL NIVEL DE COMPLEJIDAD DE ORGANIZACIÓN DE LA TOMA DE DECISIONES}

\section{RESUMEN}

En una sociedad capitalista, las decisiones de organización, por lo general destinadas a la dimensión económica, provocan o exacerban los problemas socioambientales en muchos países. En la búsqueda de soluciones a estos problemas surge en la década de i980 el concepto de desarrollo sostenible propuesto para satisfacer las necesidades de las generaciones presentes sin comprometer la atención de las necesidades de las generaciones futuras (COMISSÃO MUNDIAL SOBRE MEIO AMBIENTE E DESENVOLVIMENTO, I988). Considerado como un desarrollo subjetivo y abierto a la interpretación, la sostenibilidad incluye soporte de tres dimensiones esenciales: económica, social y ambiental. Así, bajo el punto de vista organizativo, para tomar decisiones que contribuyan a la sostenibilidad, se ha convertido por lo menos un gran desafío, porque más allá de contemplar la dimensión económica debe abordar simultáneamente las 
dimensiones social y medioambiental. Esto muestra que hay niveles de decisiones se definen como simple, promedio y complejo y que estos niveles tienden a influir o poner en peligro la sostenibilidad de manera diferente, dependiendo del servicio satisfactorio o no de las dimensiones. Ante este reto, este artículo tiene como objetivo presentar una comprensión de la sostenibilidad a través del nivel de complejidad de las decisiones de la organización. Para lograr este objetivo, este trabajo en su carácter teórico-metodológico considera los criterios pertinentes que componen cada dimensión de la sostenibilidad y la identificación de las relaciones (sistémico) que pueden ocurrir entre los criterios para la toma de decisiones de la organización. Como resultado del cruce entre los niveles de complejidad de las decisiones y las dimensiones de la sostenibilidad, se obtuvo la estructura de una matriz de los niveles de complejidad de las decisiones para abordar la sostenibilidad. En su enfoque cualitativo, la matriz tiene por objeto mostrar cómo en la toma de decisiones el investigador puede clasificar a las decisiones que considere pertinentes y cómo puede tomar decisiones complejas que se integran de manera satisfactoria a las tres dimensiones de la sostenibilidad.

\section{PALABRAS CLAVE}

Sostenibilidad; Dimensiones de la sostenibilidad; Criterios de las dimensiones de la sostenibilidad; Niveles de decisiones; Organizaciones.

\section{REFERÊNCIAS}

AMAZONAS, M. O pluralismo da economia ecológica e a economia política do crescimento e da sustentabilidade. In: ENCONTRO DA SOCIEDADE BRASILEIRA DE ECONOMIA, 8., 2009, Cuiabá. Boletim... Brasília: Ecoeco, 2009. p. 5-I2.

BERTALANFFY, L. Teoria geral dos sistemas: fundamentos, desenvolvimento e aplicações. Tradução Francisco M. Guimarães. 3. ed. Petrópolis: Vozes, 2008.

BOULDING, K. E. The economics of the coming spaceship earth. In: JARRET, H. (Ed.). Environmental quality in a growing economy. London; Baltimore: Resources for the Future; The Johns Hopkins Press, ig66.

BROWN, L. Eco-economia. [S. 1.]: Earth Policy Institute, Universidade Livre da Mata Atlântica, 2003. CAPRA, F. As conexões ocultas: ciência para uma vida sustentável. São Paulo: Cultrix, 2005. A teia da vida: uma nova compreensão científica dos sistemas vivos. São Paulo: Cultrix, 2006.

CHECKLAND, P. Soft systems methodology: a thirty year retrospective. Systems Research and Behavioral Science, v. I7, p. 5II-558, Nov. 2000.

CIEGIS, R.; RAMANAUSKIENE, J.; MARTINKUS, B. The concept of sustainable development and its use for sustainability scenarios. [S. 1.]: Inzinerine Ekonomika-Engineering Economics, 2009. 
COMISSÃO MUNDIAL SOBRE MEIO AMBIENTE E DESENVOLVIMENTO (CMMAD). NosSo futuro comum. Rio de Janeiro: Fundação Getulio Vargas, I988.

DIAS, R. Gestão ambiental: responsabilidade social e sustentabilidade. São Paulo: Atlas, 2008.

DALY, H. E. A economia ecológica e o desenvolvimento sustentável. Tradução John Cunha Comerford. Rio de Janeiro: Assessoria de Serviços e Projetos em Agricultura Alternativa, I99I. 2Ip. (Textos para debate, n. 34).

Crescimento sustentável? Não obrigado. San Francisco: Sierra Club Books, I996.

DONAIRE, D. Gestão ambiental na empresa. São Paulo: Atlas, I999.

DONAIRES, O. S. Uma abordagem sistêmica ao mapeamento e melhoria do processo de desenvolvimento de software. In: CONGRESSO BRASILEIRO DE SISTEMAS, 4., 2008, Franca. Anais... Franca: Centro Universitário de Franca, 2008.

ELKINGTON, J. Cannibals with forks: the triple bottom line of 2Ist century business. Oxford: Capstone, I999. 402p.

Enter the triple bottom line. In: HENRIQUES, A.; RICHARDSON, J. (Ed.). The triple bottom line: does it all add up. London: EarthScan, 2004.

FREITAS, J. S.; COTA JUNIOR, M. B. G.; CHENG, L. C. O soft systems thinking e a soft systems methodology: teorias, conceitos e metodologias sistêmicas. In: CONGRESSO BRASILEIRO DE SISTEMAS, 4., 2008, Franca. Anais... Franca: Centro Universitário de Franca, 2008.

GITMAN, L. J. Princípios de administração financeira. São Paulo: Harbra, 2002.

GOMES, L. F. A. M.; GOMES, C. F. S.; ALMEIDA, A. T. Tomada de decisão gerencial: um enfoque multicritério. São Paulo: Atlas, 2006.

GOODLAND, R. The concept of environmental sustainability. Annual Review of Ecology and Systematics, v. 26, p. I-24, I995.

GUIMARÃES, R. P. A ecopolítica da sustentabilidade em tempos de globalização corporativa. In: GARAY, I. E.; BECKER, B. K. (Org.). As dimensões humanas da biodiversidade: o desafio de novas relações sociedade-natureza no século XXI. Petrópolis: Vozes, 2006.

KAPP, K. W. The open system character of the economy and its implications. Tradução Dopfer. [S. 1.: s. n.], 1976.

LUZZATI, T. El desarollo ecocompatible: la atualidad la contribuición de Karl William Kapp (I9IoI976). La Revista Ecología Política en América Latina, Barcelona, v. 2, n. 30, p. 59-70, dic. 2005.

MACKNESS, J. Metodologia soft systems (SSM). In: ANDRADE, A. L. et al. (Org.). Pensamento sistêmico: caderno de campo: o desafio da mudança sustentada nas organizações e na sociedade. Porto Alegre: Bookman, 2006.

MAIA, A. G. Decisões sistêmicas em organizações turísticas: uma compreensão fenomenológica da influência dos níveis de complexidade das decisões organizacionais na sustentabilidade do turismo. 20Io. 230 f. Tese (Doutorado em Administração e Turismo)-Universidade do Vale do Itajaí, Biguaçu, 20I0.

MARTINELLI, A. C. Empresa-cidadã: uma visão inovadora para uma ação transformadora. In: IOSCHPE, E. B. Terceiro setor: desenvolvimento social sustentado. São Paulo: Paz e Terra, I997. MILLER, S. J.; HICKSON, D. J.; WILSON, D. C. A tomada de decisão nas organizações. In: CLEGG, S. R. et al. Handbook de estudos organizacionais. São Paulo: Atlas, 2004. cap. II.

MONTIBELLER FILHO, G. O mito do desenvolvimento sustentável: meio ambiente e custos sociais no moderno sistema produtor de mercadorias. Florianópolis: UFSC, $200 \mathrm{I}$. 
MORALEJO, I. A.; MIGUEL, C. E.; LEGARRETA, J. M. B. El desarrollo sostenible a lo largo de la historia del pensamiento económico. Revista del Economia Mundial, Huelva, n. 2I, p. 87-IIo, 2009. MORIN, E. Ciência com consciência. I2. ed. Rio de Janeiro: Bertrand Brasil, 2008.

MOTTA, F. C. P.; VASCONCELOS, I. F. F. Teoria geral da administração. São Paulo: Pioneira Thomson Learning, 2002.

RODRIGUES, L. H. As abordagens hard e soft. In: ANDRADE, A. L. et al. (Org.). Pensamento sistêmico: caderno de campo: o desafio da mudança sustentada nas organizações e na sociedade. Porto Alegre: Bookman, 2006.

ROGERS, P.; BLENKO, M. Quem tem a D? Como papéis decisórios explícitos aumentam o desempenho organizacional. In: HARVARD BUSINESS REVIEW. Decisões mais inteligentes. Rio de Janeiro: Elsevier, 2008.

SACHS, I. Caminhos para o desenvolvimento sustentável. Rio de Janeiro: Garamond, 2002.

SACHS, J. A riqueza de todos: a construção de uma economia sustentável em um planeta superpovoado, poluído e pobre. Tradução Sérgio Lamarão. Rio de Janeiro: Nova Fronteira, 2008.

SAMPAIO, C. A. C. Planejamento para o desenvolvimento sustentável: um estudo de caso comparativo de município. Florianópolis: Bernúncia, 2002.

SCHWANINGER, M. System dynamics and the evolution of systems movement. Systems Research and Behavioral Science, v. 23, n. 5, p. 583-594, Sept./Oct. 2006.

SHIMIZU, T. Decisão nas organizações: introdução aos problemas de decisão encontrados nas organizações e nos sistemas de apoio à decisão. São Paulo: Atlas, 200I.

SIMON, H. A behavioral model of rational choice. The Quarterly Journal of Economics, v. 69, n. I, p. 99-II8, Feb. I955.

Comportamento administrativo: estudo dos processos decisórios nas organizações administrativas. Tradução Aluízio Loureiro Pinto. Rio de Janeiro: Fundação Getulio Vargas, I97I.

TEIXEIRA JUNIOR, A. S. Temperatura, calor e energia interna. Revista de Ensino de Ciências, São Paulo, n. 6, p. 23-25, jul. I982.

VIEIRA, P. F. (Org.). Ecodesenvolvimento: do conceito à ação. In: SACHS, I. Rumo à ecossocioeconomia: teoria e prática do desenvolvimento. São Paulo: Cortez, 2007. 